\title{
Absorption Corrections for a Four-Quadrant SuperX EDS Detector
}

\author{
F. Yang ${ }^{1}$, F. Scheltens ${ }^{1}$, D. McComb ${ }^{1}$, D.B. Williams ${ }^{1}$, and M. De Graef ${ }^{2}$ \\ ${ }^{1 .}$ Dept. of Materials Science and Engineering, Ohio State Univ., Columbus OH 43210, USA \\ 2. Dept. of Materials Science and Engineering, Carnegie Mellon Univ., Pittsburgh PA 15213, USA
}

Consider a four-quadrant detector consisting of four circular active regions of area $A_{q}$ each, placed symmetrically around the sample, as shown schematically in Fig. 1(a). The sample holder is inserted from the right, and the quadrants are $90^{\circ}$ apart, oriented symmetrically at $\pm 45^{\circ}$ with respect to the primary tilt axis. The specimen tilt angles are labeled $\alpha$ and $\beta$, with positive angles corresponding to counterclockwise rotations. Each detector quadrant is located above the plane of the specimen and is tilted from the vertical plane by an angle $\delta_{d}$; the angle between the line connecting the center of a quadrant $D$ with the eucentric point $S$ and the horizontal plane is labeled $\alpha_{d}$ (Fig. 1(b)). The active circular regions have a radius of $R_{d}$, and a distance to the eucentric point of $r_{d}$; the detector opening angle is $R=R_{d} / r_{d}$. When $\bar{R}$ is not negligible, as is the case for a SuperX detector, then the x-ray photon path length inside the sample becomes a function of the position on the detector where the pho-ton hits; the absorption correction factor must thus involve an integration over the detector surface area.

The path length $\tau$ inside the sample for an x-ray photon leaving $P_{0}$ and traveling towards the point $P_{1}$ on the detector can be expressed as a fraction s of the distance between $P_{0}$ and $\mathrm{P}_{l}$ :

$$
\tau\left(x_{d}, z_{d}\right)=s\left(x_{d}, z_{d}\right) \sqrt{x_{d}^{2}+z_{d}^{2}+r_{d}^{2}} \quad \text { with } \quad s\left(x_{d}, z_{d}\right)=-\frac{\mathbf{n} \cdot \mathbf{P}_{0}}{\mathbf{n} \cdot\left(\mathbf{P}_{1}-\mathbf{P}_{0}\right)},
$$

where $\left(x_{d}, \mathrm{z}_{\mathrm{d}}\right)$ are the in-plane detector coordinates of the point $P_{0}$. Using the quadrant numbering of Fig. 1(a), one can show that the thickness-integrated dimensionless absorption correction factor for quadrant $i$ is given by:

$$
C_{i}(\mu t)=\left[\frac{1}{\pi \bar{R}^{2}} \iint_{D} \mathrm{~d} \bar{x} \mathrm{~d} \bar{z} \frac{\left.1-e^{-\mu t f_{i}(\bar{x}, \bar{z}}\right)}{\mu t f_{i}(\bar{x}, \bar{z})}\right]^{-1},
$$

where

$$
f_{i}(\bar{x}, \bar{z})=\frac{\sqrt{\bar{x}^{2}+\bar{z}^{2}+1}}{a_{i} \bar{x}+b_{i} \bar{z}+c_{i}}
$$

with

$$
\left\{\begin{array}{l}
a_{i}=s_{i} \sin \alpha \cos \beta+c_{i} \sin \beta \\
b_{i}=\cos \delta_{d} \cos \alpha \cos \beta+\sin \delta_{d}\left(c_{i} \sin \alpha \cos \beta-s_{i} \sin \beta\right) \\
c_{i}=\sin \alpha_{d} \cos \alpha \cos \beta-\cos \alpha_{d}\left(c_{i} \sin \alpha \cos \beta-s_{i} \sin \beta\right),
\end{array}\right.
$$

and $\bar{x}=x / r_{d}$ and $\bar{z}=y / r_{d}$. This integral poses no numerical difficulties, as the integrand is well behaved, even when the denominator of $f_{i}$ vanishes (in which case the integrand equals unity). In the absence of sample tilt ( $\alpha=\beta=0$ ), the expression for $f_{i}$ reduces to

$$
f_{i}(\bar{x}, \bar{z})=\frac{z \sqrt{\bar{x}^{2}+\bar{z}^{2}+1}}{\cos \delta_{d} \bar{z}+\sin \alpha_{d}},
$$

which, in the limit of vanishing detector radius $R_{d}$, becomes

$$
f_{i}=\frac{z}{\sin \alpha_{d}}=z \csc \alpha_{d}
$$


i.e., the standard result for a small distant detector. Since the four quadrant detectors are located symmetrically with respect to the specimen we have the following symmetry relations between the correction factors:

$$
C_{2}(\alpha, \beta ; \mu t)=C_{1}(\alpha,-\beta ; \mu t), \quad C_{3}(\alpha, \beta ; \mu t)=C_{1}(-\alpha,-\beta ; \mu t), \quad C_{4}(\alpha, \beta ; \mu t)=C_{1}(-\alpha, \beta ; \mu t),
$$

so that we only need to compute $C_{1}(\alpha, \beta ; \mu t)$ over the entire range of tilt angles. Fig. 2 shows a contour plot of the inverse correction factor $1 / C_{1}(\alpha, \beta ; \mu t)$ as a function of tilt angles $(\alpha, \beta)$ and for the detector parameters indicated in the upper left corner.

\section{References}

[1] MDG was supported by the Air Force Office of Scientific Research, MURI contract \# FA9550-12-1-0458.
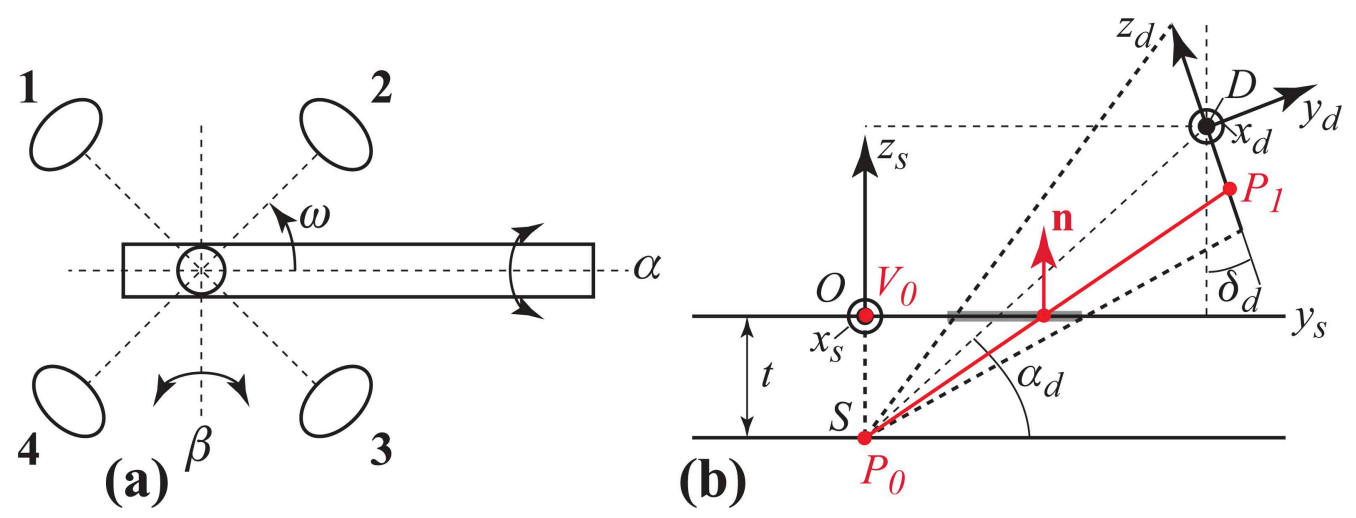

Figure 1. (a) Schematic representation of a double tilt sample holder and four quadrant detectors placed symmetrically around the specimen; (b) Schematic of the specimen with thickness $t$, and a single circular quadrant detector

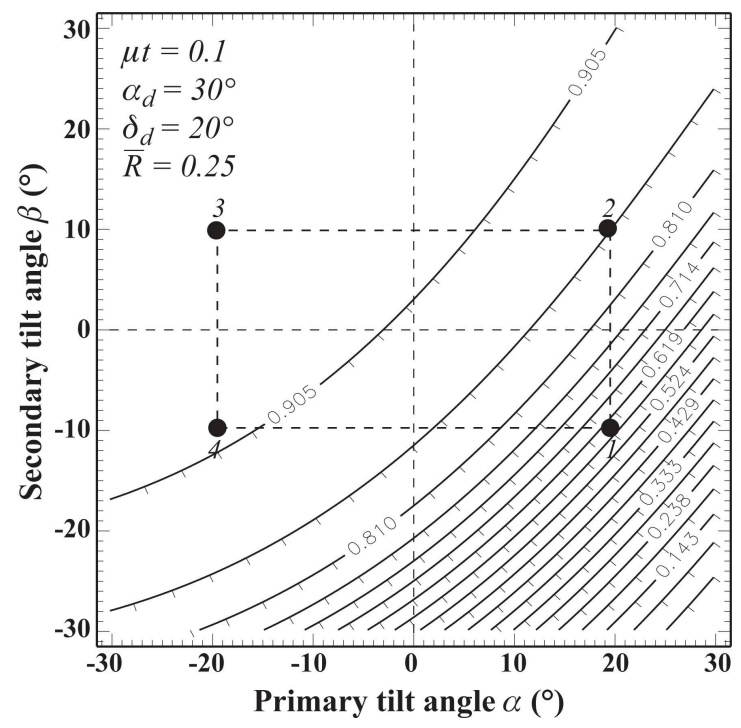

Figure 2. Contour plot of $1 / C_{1}(\alpha, \beta ; \mu t)$ for the detector parameters indicated in the upper left corner. The rectangle superimposed on the plot shows the symmetry relations between an arbitrary pair of angles $(\alpha, \beta)$ and the corresponding points for the other three detector quadrants. 